\title{
Sex-Dependent Effects of Prenatal Stress on Learned Helplessness and Anxiety-Related Behaviours in Wistar Rats
}

\author{
Inês Pereira-Figueiredo1, Consuelo Sancho'1,2,3, Juan Carro' ${ }^{1,4}$, Dolores E. López ${ }^{1,2,5}$, \\ Ricardo Gómez-Nieto ${ }^{1,2,5}$, Orlando Castellano ${ }^{1,2,5^{*}}$ \\ ${ }^{1}$ Neuroscience Institute of Castilla y León, University of Salamanca, Salamanca, Spain \\ ${ }^{2}$ Institute of Biomedical Research of Salamanca, University of Salamanca, Salamanca, Spain \\ ${ }^{3}$ Department of Physiology and Pharmacology, University of Salamanca, Salamanca, Spain \\ ${ }^{4}$ Department of Basic Psychology, Psychobiology and Methodology, University of Salamanca, Salamanca, Spain \\ ${ }^{5}$ Department of Cell Biology and Pathology, University of Salamanca, Salamanca, Spain \\ Email: ${ }^{*}$ orlandoc@usal.es
}

Received 10 June 2015; accepted 7 July 2015; published 10 July 2015

Copyright (C) 2015 by authors and Scientific Research Publishing Inc.

This work is licensed under the Creative Commons Attribution International License (CC BY). http://creativecommons.org/licenses/by/4.0/

(c) (i) Open Access

\begin{abstract}
There has been an increasing importance of studies that link sex to stress coping processes. Recently, we reported that male and female Wistar rats responded differently to prenatal stress (PS) under basal conditions. The aim of the present study was to determine the influence of sex on behaviour and coping strategies, as an effect of gestational adversity in rats that were exposed to an uncontrollable stressor. Once the animals reached adulthood, the offspring from stressed/nonstressed dams were subjected or not to antidepressant treatment with Sertraline. After that, they were exposed to a single inescapable shock (IS) session, in which the rats were further tested for escape behaviour along 10 days, as a model of learned helplessness (LH). In prenatally stressed animals after the IS, behavioural differences appeared in a sex specific manner. Males proved to be more susceptible to the adverse context than females, exhibiting behavioural despair in a large percentage of the cases. Surprisingly, PS did not affect shock escape failure, but did affect learning performance in a sex dependent manner. In females, PS led them to learn to avoid shocks, learning better than controls, and by contrast, PS males did not learn to avoid shocks and displayed some signs of anhedonia. Sertraline did not help animals to avoid shocks, but helped them to escape from it. Our data indicate the existence of sex dependent behavioural differences in PS animals when facing an uncontrollable stress situation, in which the changes induced by PS were not only different, but opposite between sexes.
\end{abstract}

*Corresponding author.

How to cite this paper: Pereira-Figueiredo, I., Sancho, C., Carro, J., López, D.E., Gómez-Nieto, R. and Castellano, O. (2015) Sex-Dependent Effects of Prenatal Stress on Learned Helplessness and Anxiety-Related Behaviours in Wistar Rats. Journal of Behavioral and Brain Science, 5, 251-265. http://dx.doi.org/10.4236/jbbs.2015.57026 
Keywords

Gestational Stress, Gender Differences, Sertraline, Escape Behaviour, Inescapable Footshock

\section{Introduction}

It is currently established that early adverse experiences cause changes in the nervous system that persist throughout life [1] [2]. Such experiences have a high impact as regards the future predisposition of an individual's reactivity and propensity to suffer from psychological illnesses [3] [4]. In pregnant females, prenatal stress (PS) is an adverse experience known to enhance developmental disorders and to elicit several psychopathologies associated with anxiety or depression in newborns [5]-[8]. The majority of the studies using prenatal stress animal models were carried out in males without any further investigations into sex-related behavioural differences [3] [9] [10]. Even though early reports on the sex variation in response to early stressors were inconsistent [11], more recent studies addressing prenatal stress in several species have confirmed the existence of sex-dependent factors [2] [12]-[14] that are responsible for the divergent epigenetic changes in males and females. This suggests the possibility that early stressors might elicit sex-specific effects on later vulnerability to stress in the offspring.

Overall, animals that are exposed to inescapable or uncontrollable stress frequently develop learned helplessness (LH) [15]. This condition reflects the despair they possibly feel when they have no control over their circumstances, and it has been proposed as a model of the "stress and coping" paradigm [15] [16]. It is considered that certain forms of human depression are shared with LH animals and it has been suggested that the underlying mechanism is similar [17] [18]. Both, humans and animals show an apparent lack of interest in their environment as they feel their actions are meaningless, and that there is a lack of congruence between actions and consequences, which might have key medical implications. Moreover, this phenomenon is sensitive to antidepressant administration [19] [20]. Thus, in the present study, our aim is to determine what happens to animals previously exposed to inescapable shocks (IS) when they are re-exposed to the same box. Our study attempts to determine whether these animals will exhibit contextual fear and escape deficits and whether chronic treatment with sertraline (SERT), a selective serotonin reuptake inhibitor (SSRI) known to reverse depressive symptoms [20], will counteract its effects. Recently, we have reported that the administration of SERT along adolescence is safe in rodents, and reverses some of the prenatal stress effects. Thus, in the present study this drug was administered during the same period to rats. In this sense, we analyzed prenatal stress-induced changes in the later behaviour of the offspring of stressed mothers, focusing on the potential differences between sexes both, as regards stress vulnerability and the impact of chronic treatment with SERT, as a modulating factor.

\section{Materials and Methods}

\subsection{Animals}

Virgin female Wistar rats CLS: WI (HAN) $(\mathrm{n}=12)$ weighing $250 \mathrm{~g}$ were obtained from highly out bred rats from the animal facility at the University of Salamanca. Vaginal smears were collected daily for 8 days before mating to determine the stage of the oestrus cycle and the day of conception. On the day of proestrus, sexually experienced male Wistar rats were introduced for mating. The day on which spermatozoa were found in the smear was designated as day 1 of pregnancy. For all experiments, the animals were allowed access to food and water ad libitum, and were maintained on a regular light-dark cycle (lights on: 07:00 to 19:00 h) in a temperature- and humidity-controlled environment. The animals were handled and cared for according to the guidelines of the European Community's Council Directive (2010/63/EU), current Spanish legislation (RD 1201/05), and those established by the Institutional Bioethics Committee for the care and use of laboratory animals.

\subsection{Prenatal Stress Exposure}

Pregnant female rats were randomly assigned to the stress or control groups ( $\mathrm{n}=6$ per group) and housed individually in plastic breeding cages. Stress consisted of placing females in the third trimester (days 15 - 21 of gestation) in transparent cylindrical restrainers (7 cm diameter, $19 \mathrm{~cm}$ long); under a bright light (50 W bulb) di- 
rected onto the surface of the restrainer for 45 min three times a day (at 09:00, 12:00 and 16:00 h). Control mothers were left undisturbed, only being handled for routine activities (cleaning, etc.). Only offspring from litters containing 9 - 12 pups were used in the present experiments. Offspring were weaned at 21 days of age and were separated into group cages housing four animals of the same sex and treatment as previously described [13]. No differences in litter sizes, in the male-to-female ratio of the offspring or inpre-weaning-mortality were found.

\subsection{Drug Administration}

Starting at postnatal day 30 (P30) and continuing until the end of experiments (P115), pups from each condition-Control or PS depending on the previous treatment-were subdivided to receive either chronic treatment with SERT (Control-SERT and PS-SERT) or saline (Control, PS) ( $n=8$ per sex and group). Based on previous reports [13], we decided to administer SERT orally (Besitran ${ }^{\circ}$ Pfizer S. A. Madrid, Spain) at a dose of 5.0 $\mathrm{mg} / \mathrm{kg} /$ day. Liquid consumption was controlled by using calibrated bottles, and was monitored every two days, and the dose of the drug was adjusted on the basis of the liquid consumed. During this period, the rats were kept in groups of 4 animals in polycarbonate boxes $(45 \times 30 \times 20 \mathrm{~cm})$, with unrestricted access to food.

\subsection{Inescapable Shocks}

Over 2 days before testing, the animals were habituated to the experimental conditions by being placed in the apparatus and left undisturbed for 3 minutes. The shuttle box used to administer the footshocks had two equal chambers $(50 \times 25 \times 25 \mathrm{~cm})$ separated by a black Plexiglas partition with a gate that could be opened or closed (Letica Scientific Instruments, Spain). In this test, footshocks were administered in the left chamber with the gate closed. The floor consisted of a stainless-steel bars set $2 \mathrm{~cm}$ apart, connected in series to a main control module (Letica, LI-2900, Spain) through which the electric shocks were administered. The rats were placed individually in the experimental chamber and after 1 min of acclimatization, a sequence of 3 electric footshocks ( $0.35 \mathrm{~mA}, 5 \mathrm{~s}$, with $20 \mathrm{~s}$ between each one) were given, with no warning light signal either before or during each shock. After another minute of rest, the rats were returned to their home cages. The sensitivity to shocks was assessed by the occurrence of jump reactions and audible vocalizations, both as measures of physical integrity [21] [22]. The value of each shock vocalization response was classified as no response, when no audible sound was detected (corresponding to a value of 0 ); a mild response, the emitted sound lasted less than a second (corresponding to a value of 0.5); and a strong response, when the emitted sound last more than one second (corresponding to a value of 1). The total time spent freezing, as seen by a period of at least 4 consecutive seconds with no visible movements, including the vibrissae, was also assessed along the sessions. All observations were carried out in a single-blind assessment by two different investigators.

\subsection{Escapable Shocks}

Four days after the IS, all animals were re-exposed to the same shuttle box used previously to test their escape/ avoidance performance. During this test, the gate was opened to allow free movement between the compartments. Thus, the animals were able to learn that they could escape the shocks. Both compartments of the box were equipped with a light source $(15 \mathrm{~W}$ )-located on the upper lateral wall and connected to an electrifiable bar-floor - that was used as the conditioned stimulus (CS). The delivery of footshocks, unconditioned stimulus (US), in both compartments was independent; the floor was hinged to operate a switch when depressed, allowing the responses to be controlled automatically. Rats were tested in 10 consecutive daily sessions, each session with 10 trials. The animals were placed in the left chamber of the shuttle box and allowed to explore for $30 \mathrm{~s}$, before the first trial began. Each sequence of 10 trials consisted of a $5 \mathrm{~s}$ warning signal with the light on, followed by $3 \mathrm{~s}$ of $0.25 \mathrm{~mA}$ electric shock and 30s of inter-trial time. Crossing into the opposite chamber while the light signal was on was counted as avoidance, and crossing the chamber while receiving the electric shock was counted as escape. The shock and light signal were terminated immediately once the rat had moved to the other chamber. A non-crossing response during shock delivery was considered escape failure [23] and the rat received a shock lasting $3 \mathrm{~s}$. The criterion for conditioning was the observation of 2 consecutive sessions with more than $70 \%$ avoidance responses, and an extreme behavioural response was considered when the animals failed to escape in 3 consecutive sessions (with 100\% of non-crossing responses). The movements through the gate during 
the $30 \mathrm{~s}$ prior to each session were counted as a crossing during adaptation and the movements during the session with no stimulation, were counted as inter-trial crossings [24]. Both were used as indicators of locomotor activity under the experimental condition. The number of faecal boluses, and the reactions to CS (light) and US (shocks) were also assessed throughout the sessions.

The oestrous cycle was not determined in order to prevent differential manipulation of the male and female rats. In light of the previous studies [17] [25] the despair behaviour or the escape latency upon using LH tests is not influenced by the different phases of oestrus.

\subsection{Statistical Analysis}

Statistical analyses were performed using the SPSS software, version 18.0 (SPSS Inc., Chicago, IL, USA). The differences between groups were analyzed by analysis of variance (one, two, and three way ANOVA), followed by the Fisher-PLSD-test for post-hoc comparison if appropriate, and ANOVA mixed ("SPLIT-PLOT") with the Bonferroni test. Differences between groups were regarded as statistically significant when $p<0.05$. Pearson's coefficient was used to determine correlations. All values are expressed as mean values \pm standard error (S.E.M.)

\section{Results}

\subsection{Inescapable Footshocks}

The sensitivity to footshock presentation was assessed by the responses of jumps and vocalizations (Figure 1). An effect of PS was revealed in the number and intensity of vocalizations $\left(\mathrm{F}_{3.56}=7.38, p<0.001\right)$. Prenatally stressed animals of both sexes vocalized less in their reaction to shocks than controls (Figure 1). In both sexes, SERT acted by reversing the effect of early stress on decreasing the response to shocks. Regarding the jump reaction, differences between sexes were found to affect the animals' response to footshocks $\left(F_{1.56}=9.8, p=\right.$ 0.003). Within non-treated animals, the females responded more intensely than males $(p<0.01)$. In females, a main effect group $\left(\mathrm{F}_{3.28}=4.9, p=0.008\right)$ was found, once SERT decreased the jump reaction to shocks (Figure 1). In males, again, early stress was the only factor affecting this measure; PS males responded less than the controls, although the difference was not significant $(p=0.47)$.

Moreover, the analysis of the period of time animals spent freezing during the footshock session revealed a main effect of group $\left(\mathrm{F}_{3.56}=5.7, p=0.002\right)$, with a sex vs. group interaction $\left(\mathrm{F}_{3.56}=6.12, p=0.001\right)$. An overall

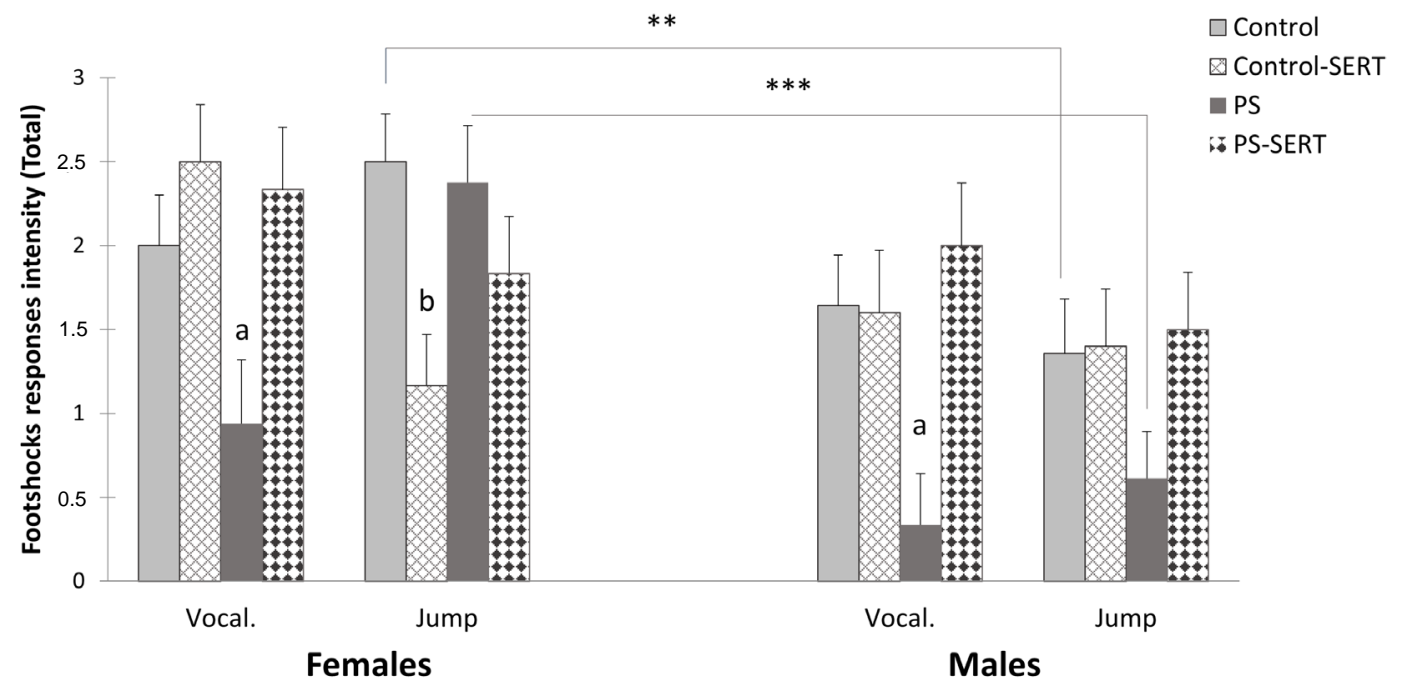

Figure 1. Effects of prenatal stress and SERT treatment on footshocks reaction. It is shown the effects of prenatal stress and SERT treatment $(5 \mathrm{mg} / \mathrm{kg} /$ day $)$ on footshocks reaction in the animals of both sexes. a: $p<0.05$, indicates a main effect of prenatal stress; b: $p<0.05$, indicates a main effect of SERT; ${ }^{* *} p<0.01 ;{ }^{* * *} p<0.001$, indicate a main effect of sex within non treated animals. Each bar represents the sum of the 3 footshocks presentation intensity, expressed as means \pm S.E.M. 
effect of SERT in decreasing the freezing response to shocks was found in both sexes; but also differences according to sex are shown, as an effect of the early stress $(p<0.001)$ (Figure 2). Post-hoc reveals that PS females freeze more than controls $(p=0.06)$; otherwise, PS males displayed significantly less time freezing than their control counterparts $(p<0.001)$ (Figure 2).

\subsection{Escapable Footshocks}

\subsubsection{Avoidance Behaviour}

Differences were found depending on group $\left(\mathrm{F}_{3.49}=5.7, p=0.002\right)$, and $\operatorname{sex}\left(\mathrm{F}_{1.49}=8.6, p=0.005\right)$, and a sex group $\left(\mathrm{F}_{3.49}=3.18, p=0.032\right)$ interaction was observed.

A mixed-factor ANOVA for sex vs. treatment vs. sessions revealed an overall increase in avoidance behaviour along the daily sessions $\left(\mathrm{F}_{9.441}=5.7, p<0.001\right.$ ) (Figure 3(A) and Figure 3(B)). When each group was compared with its pair-matched control, post-hoc analysis revealed sex differences for the prenatally stressed and Control-SERT animals; the females exhibited a higher number of avoidance responses than the males $(p<$ 0.001 in both groups). Among the females, post-hoc analysis revealed that the PS females developed the greatest ability in learning to avoid adverse stimuli (PS vs. Control $+15.2 \% \pm 3.9, p=0.001$ ). In males, prenatal stress did not affect the acquisition of avoidance behaviour (Figure 3(A) and Figure 3(B)). SERT administration affected the learning avoidance of the shocks differently in controls and PS animals (Figure 3(C)). In control females, SERT administration helped the learning avoidance, an effect that, from the $6^{\text {th }}$ session reached significance $(p=0$. 018) (Figure 3(A)). Paradoxically, in prenatally stressed females, an effect of the drug disrupting such behaviour was found (Figure 3(A)). Also, the males receiving SERT showed the poorest avoidance performance (Figure 3(B)). On comparing the percentage of animals in each group that reached the conditioning criterion, differences were also found: the prenatally stressed females obtained the highest success rate of avoidance, $87.5 \%$ of PS females fulfilled the proposed criterion vs. $44.4 \%$ of Controls; $66.6 \%$ of Control-SERT; and $16 \%$ of PS-SERT. In males, neither prenatal stress (22 \% males PS vs. $14 \%$ of Control males) nor SERT treatment (16\% of PS-SERT vs. $16 \%$ of males Control-SERT males) affected the percentage of animals reaching the conditioning criterion (data not shown).

\subsubsection{Escape Behaviour}

The analysis of escape behaviour (sex vs. group vs. sessions) revealed a significant change $\left(\mathrm{F}_{9.441}=8.43, p<\right.$ $0.001)$, with a group interaction $\left(\mathrm{F}_{27.441}=2.26, p<0.001\right)$, probably due to the effect of SERT on this parameter in both sexes $\left(\mathrm{F}_{3.49}=23.2, p<0.001\right)$. The animals receiving SERT displayed significantly more escape responses than their control counterparts (Figure 4). Among the males (Figure 4(B)), the differences reached significance in both groups of SERT-taking animals, regardless of the previous stress treatment. In females (Figure 4(A)), the differences reached significance in previously stressed animals. Although in the first three sessions, Control-SERT females also exhibited significantly more escape responses than controls, there was a moment as from which this response decreased in the Control-SERT females (Figure 4(A)).

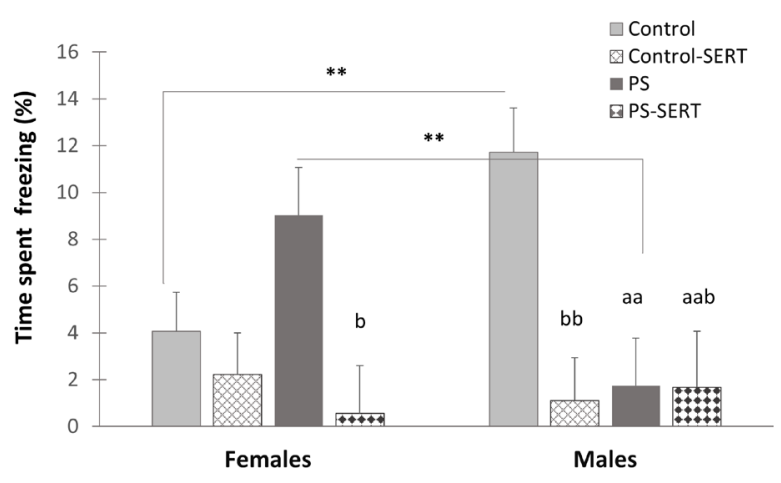

Figure 2. Effects of prenatal stress and SERT treatment on the time rats spent freezing throughout the footshocks session. You may see the effects produced by prenatal stress and SERT treatment $(5 \mathrm{mg} / \mathrm{kg} / \mathrm{day})$ on the time the rats spent freezing-expressed in percentage-throughout the footshocks session. aa: $p<0.01$, indicates a main effect of prenatal stress in males; bb: $p<0.01$; b: $p<0.05$, indicate a main effect of SERT; aab: $p<0.01$, indicate differences between PS-SERT and Controls. ${ }^{* *} p<0.01$; indicate a main effect of sex within non treated animals (mean values \pm S.E.M.). 


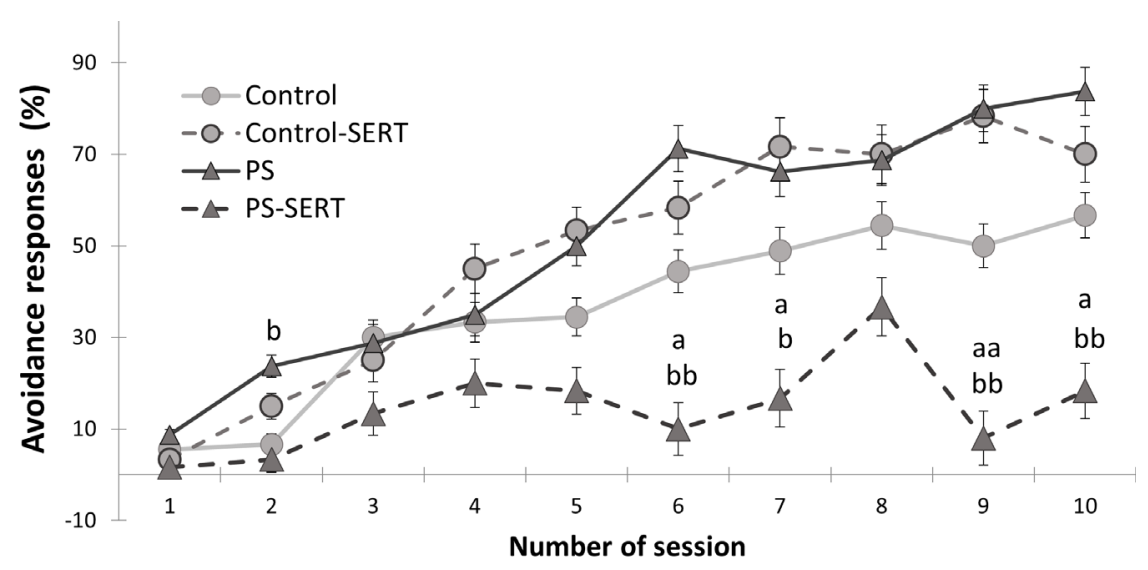

(A)

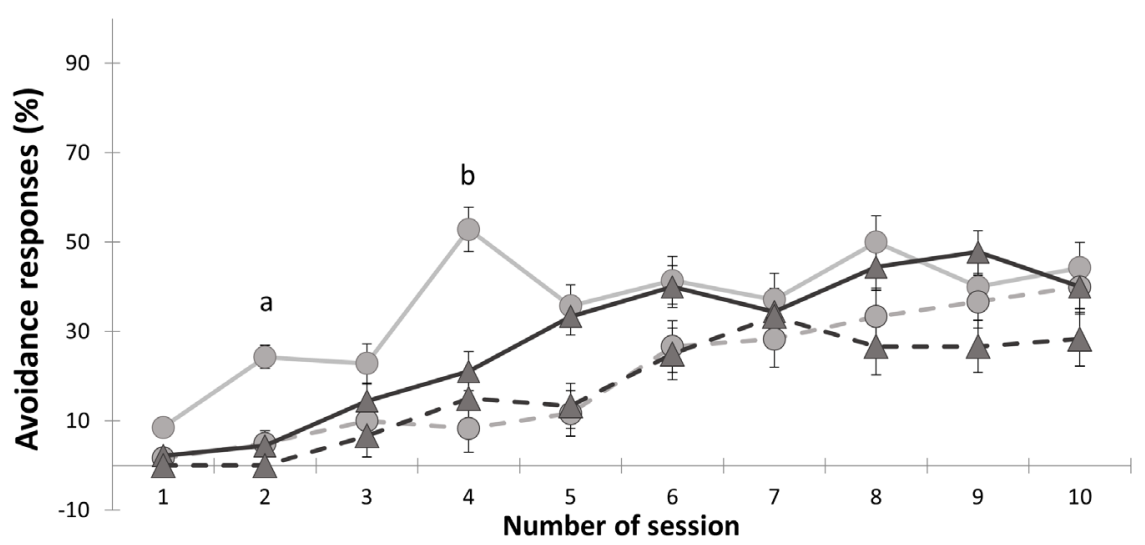

(B)

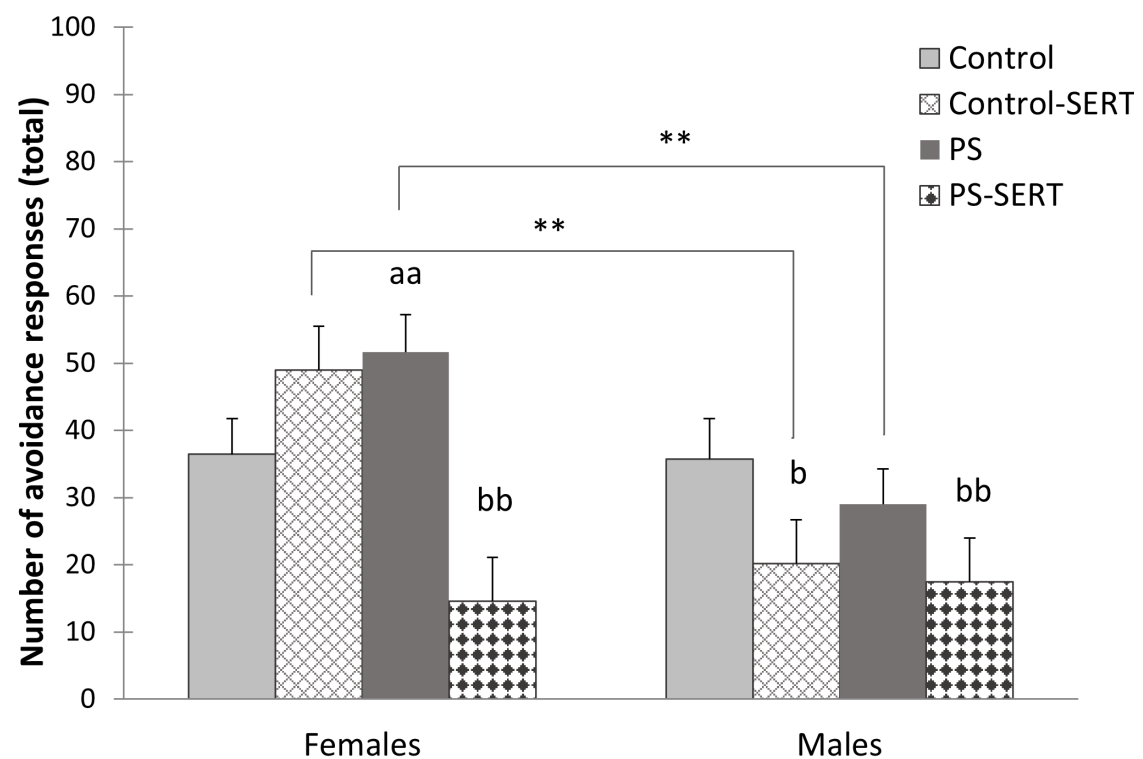

(C)

Figure 3. Acquisition of the avoidance responses. Avoidance responses all over the daily sessions in females (A) and males (B), in each experimental group; and (C) comparison between males and females in each of the experimental groups (each bar represents the sum of avoidance responses in the ten daily sessions). $\mathrm{n}=7-9$ per group and sex. a: $p<0.05$ and aa: $p<0.01$ indicate a main effect of prenatal stress; b: $p<0.05$ and bb: $p<0.01$, indicate a main effect of SERT. ${ }^{* *} p<0.01$; indicate a main effect of sex (mean values \pm S.E.M.). 


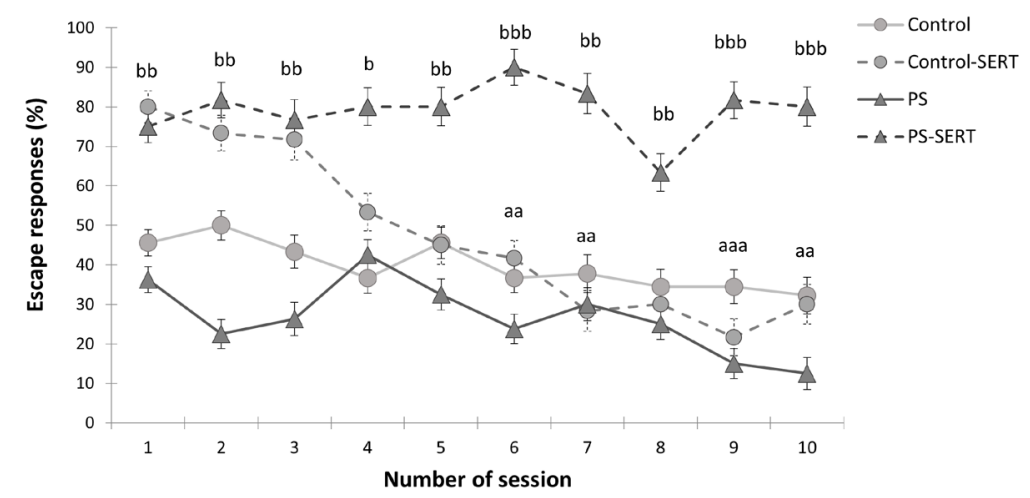

(A)

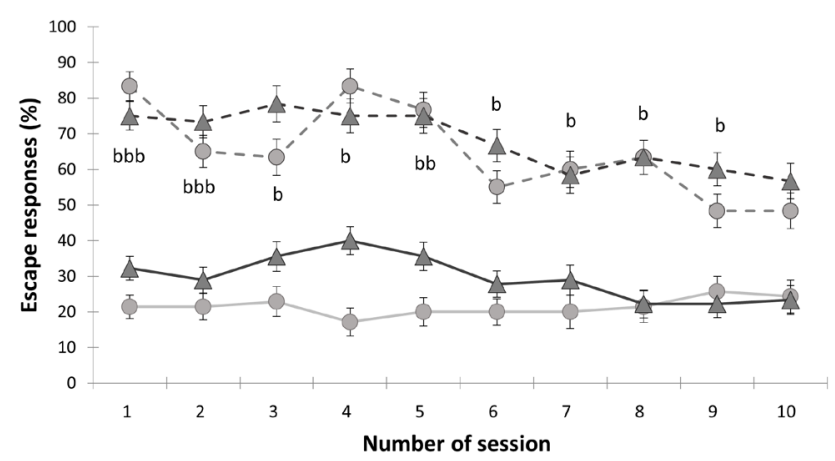

(B)

Figure 4. Acquisition of the escape responses all over the daily sessions. Escape responses in females (A) and males (B), in each experimental group. $\mathrm{n}=7-9$ per group and sex. aa: $p<0.01$ and aaa: $p<0.001$ indicate a main effect of prenatal stress; b: $p<0.05$, bb: $p<0.01$ and bbb: $p<0.001$, indicate a main effect of SERT (mean values \pm S.E.M.).

\subsubsection{Escape Failure}

An overall decrease in escape failure along the training sessions was found $\left(\mathrm{F}_{9.441}=17.23, p<0.001\right)$, with a group interaction $\left(\mathrm{F}_{9.441}=1.6, p=0.03\right)$, probably due to the effect of SERT treatment $\left(\mathrm{F}_{3.49}=14.2, p<0.001\right)$, on prenatally stressed or Control animals in both sexes. SERT acted, by helping the animals to escape from shocks (Figures 5(A)-(C)). The SERT-treated animals of both sexes exhibited significantly fewer non-crossing responses than the untreated rats (Figure $5(C)$ ), regardless of previous stress. Within the untreated animals, all of them began by failing to escape the shocks, but in the following sessions-during which the females began to avoid orescape from the shocks (Figure 5(A)) - the males, regardless of stress, exhibited escape failure and failed to respond at all (Figure 5(B)). Thus, differences between males and females were observed, the males displaying significantly more non-crossing responses than females $(p<0.05)$ (Figure 5(C)).

\subsubsection{Inter-Trial Activity}

No significant differences between the experimental groups or sexes were found upon changing the activity before the beginning of the session (crossing in adaptation). By contrast, the number of inter-trial crossings was affected by group $\left(\mathrm{F}_{3.49}=4.3, p=0.009\right)$, with a group vs. sex interaction $\left(\mathrm{F}_{3.49}=3.83, p=0.015\right)$. In both sexes, previously stressed animals receiving SERT administration showed significantly fewer crossings than the animals from the other experimental groups (Figure 6). Also, in prenatally stressed animals a main effect of sex, $\mathrm{F}_{1.49}=6.4, p=0.015$, was observed. Whereas the PS males did not show any motivation to cross the gate, PS females displayed more crossings than the controls $(p=0.04)$. The increase in inter-trial activity was positively correlated with the avoidance responses $\left(r=0.91^{*}\right)$, meaning that the more active they were during the test, by passing from one compartment to the other by trial and error, the more the rats learned to associate the stimuli. By contrast, this parameter was negatively related to the non-response level $\left(r=-0.6^{*}\right)$. 


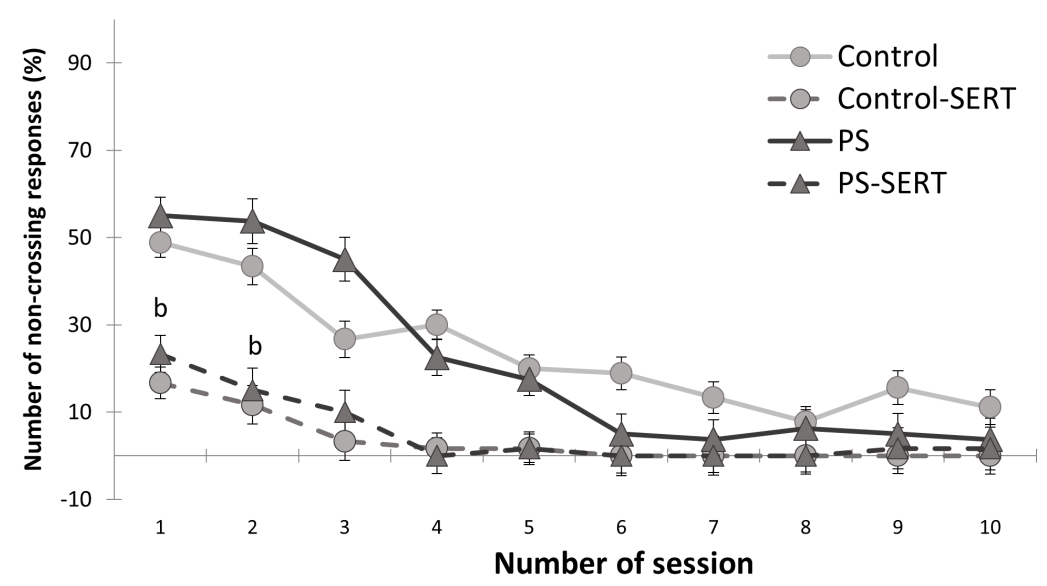

(A)

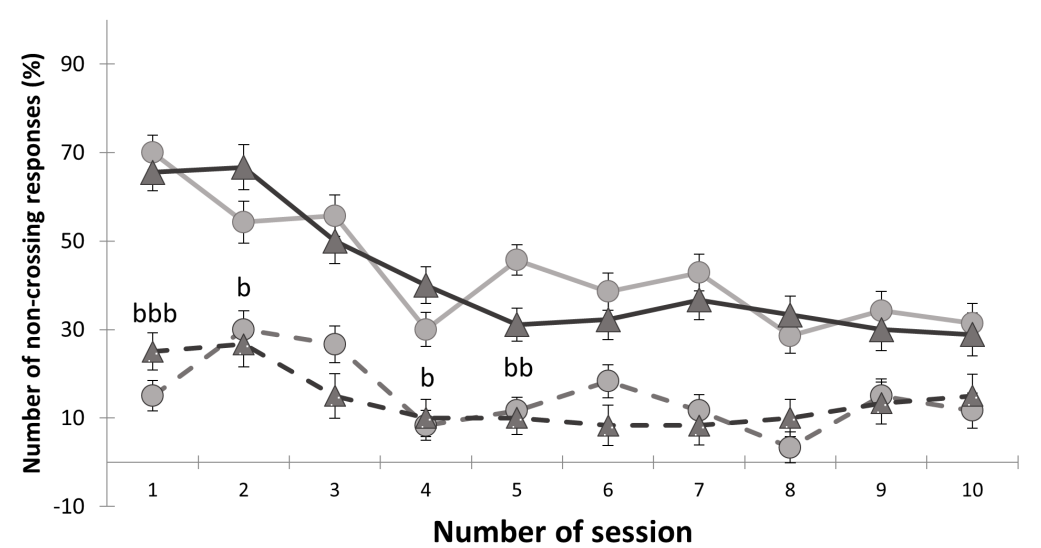

(B)

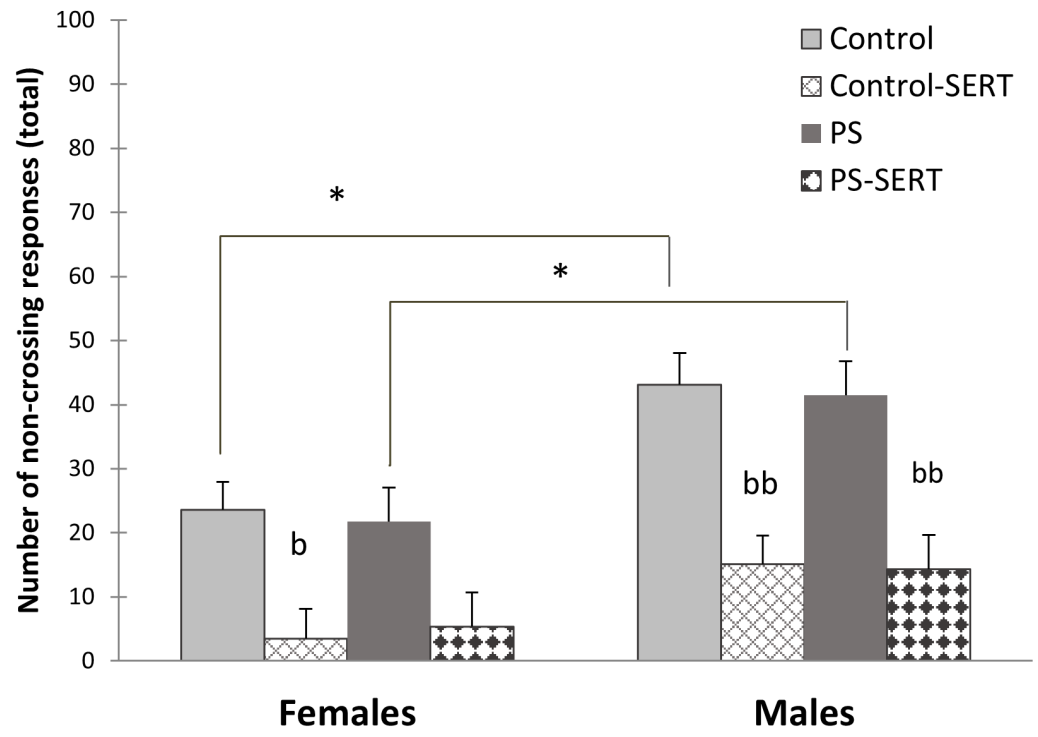

(C)

Figure 5. Effects of SERT on prenatally stressed or control animals in the escape failure. Escape failures all over the daily sessions, in females (A) and males (B) in each experimental group; and (C) comparison between males and females in each of the experimental groups (each bar represents the sum of escape failures in the ten daily sessions). $\mathrm{n}=7$ - 9 per group and sex. b: $p<0.05$ and bb: $p<0.01$, indicate a main effect of SERT. ${ }^{*} p<0.05$ indicates a main effect of sex within non treated animals (mean values \pm S.E.M.). 


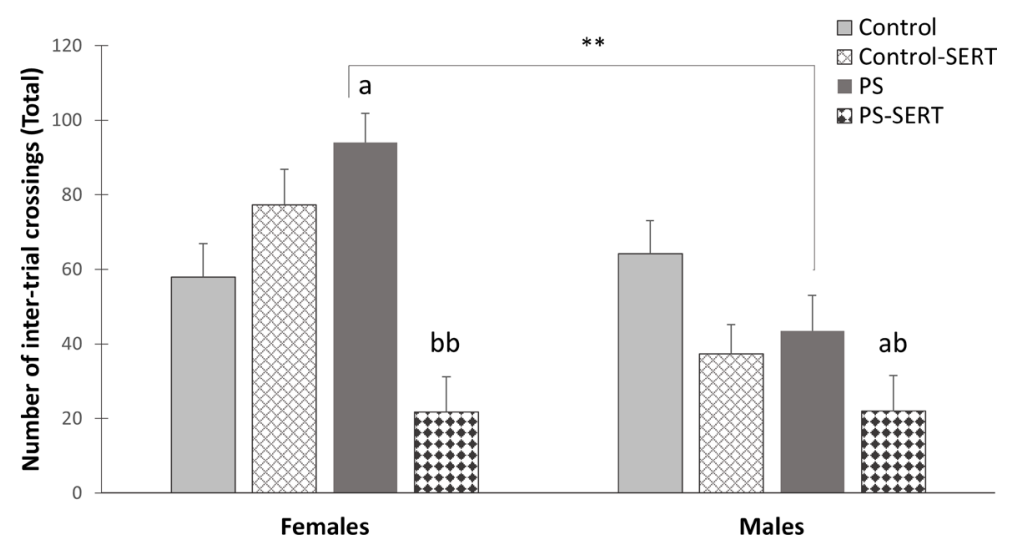

Figure 6. Inter-trial activity performed by the animals. Inter-trial activity exhibited by both sexes in each experimental group. a: $p<0.05$ indicates a main effect of prenatal stress; ab: $p<0.05$ indicates differences between the PSTF and Control males; bb: $p<0.01$, indicates a main effect of SERT; ${ }^{* *} p<0.01$; indicate a main effect of sex in prenatally stressed animals. Each bar represents the sum of inter-trial crosses all over the ten escapable footshocks sessions (mean values \pm S.E.M.).

\subsubsection{Emotionality Measures}

Throughout testing, differences between groups in the number of faecal boluses were revealed $\left(\mathrm{F}_{3.49}=20.9, p<\right.$ 0.001). When exposed to the footshocks' box the rats of both sexes receiving SERT exhibited an increase of the faecal boluses (Figure 7(A)); however, no effects of PS nor differences between the sexes were found. The responses to the stimulus were also counted (the footshocks as the US; and the light source as the CS). Throughout the escapable shock sessions, neither treatment nor sex affected the response to the footshocks (the vocalizations and jump reflex number). However, looking towards to the light source was dependent on the treatment $\left(\mathrm{F}_{27.441}\right.$ $=2.67, p<0.001$ ) (Figure 7(B)). Once again, in both sexes the animals receiving SERT exhibited more responses to stimuli than the untreated animals.

\section{Discussion}

According to the literature, individuals' responses to stressors or their propensity to suffer from affective disorders is partly based on their experiences early in life [1] [4] [5] [8]; sex-dependent differences remain to be elucidated. The main findings of the present study indicate that the restraint stress applied to rat mothers during the last week of their pregnancies acts differently in males and females as regards to their ability to respond under a stressful condition. After being exposed to uncontrollable shocks, all animals exhibited learning deficit when re-exposed to the fear context; the exceptions were PS females. These results extend recent findings reporting that rather than being unfavourable, mild early-life stress may help animals to adapt better to a stressful context later on in life [10] [26]. However, the differences according to sex must be taken into account. Furthermore, despite no particular differences in escape failure were found as an effect of PS in both sexes, the PS males exhibited signs of anhedonia, responding less to footshock delivery. SERT has shown to be effective in reversing the LH in both sexes, but increased anxiety in each exposure to the fear context.

\subsection{Inescapable Shock Reaction}

With footshock administration, in the present study, all animals responded with vocalizations or jumps and freezing. These types of reaction are considered important defensive reflexes that may help to protect the animal from injury [21] [27] [28]. Our data show that prenatal stress affected the footshocks response, especially in males. Prenatally stressed males jumped, vocalized and froze less when exposed to the shocks, in comparison with their controls. In both sexes, SERT also induced a decrease in the time spent freezing. It is well documented that there are fundamental dimorphic sex differences at the level of the brain structures and in neurotransmitter systems [29]. In the present study, when subjected to footshocks, the females were consistently more reactive than males (jumped significantly more), while the males froze more often, in complete agreement with the literature [22] [27]. Furthermore, it has been suggested that the nociceptive system of offspring may be 


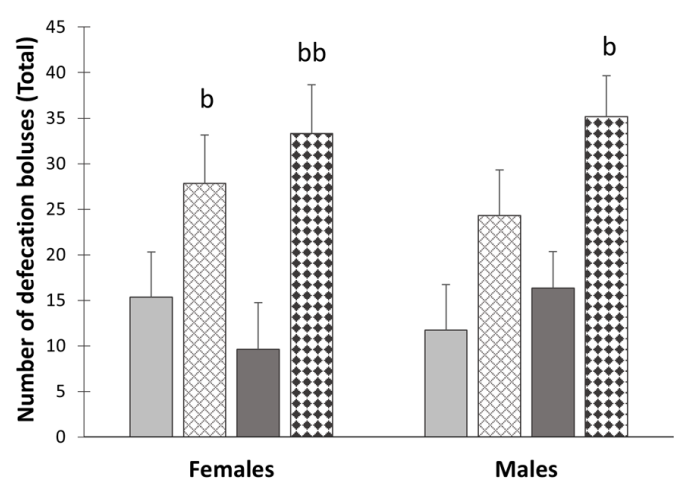

(A)

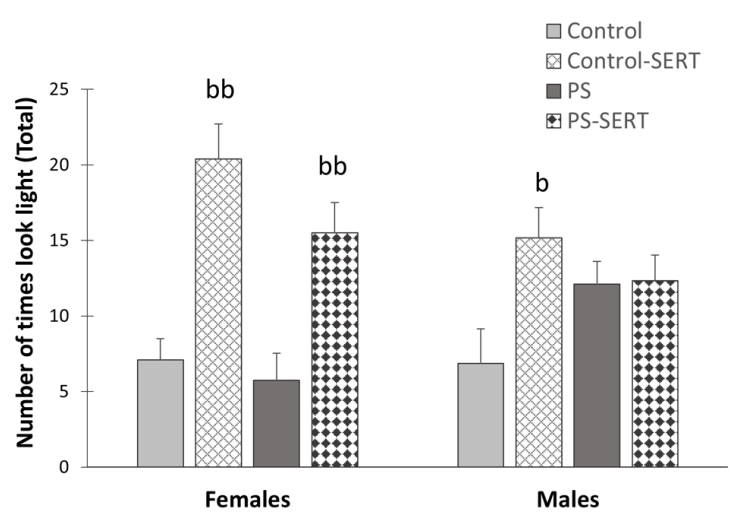

(B)

Figure 7. Effects of prenatal stress and SERT treatment on emotionality measures. Prenatal stress and SERT treatment $(5 \mathrm{mg} / \mathrm{kg} /$ day)effects in the animals of both sexes when repeatedly exposed to the shuttle box previously paired with Inescapable footshocks. bb: $p<0.01$ and $\mathrm{b}: p<0.05$, indicate a main effect of SERT. Each bar represents the sum of boluses (A) and the light orientation responses (B) in the ten daily sessions (mean values \pm S.E.M.).

altered by exposure to early stressors. The endogenous opioid changes, induced by a stressful pregnancy, can significantly affect the mediation of the nociceptive systems in the offspring that may be different in each sex [30] [31]. In fact, it could be proposed that prenatally stressed males exhibit a lower sensitivity to shocks, which SERT reversed. However, when the shocks reaction were examined later (throughout the escapable shock procedure), no differences were found. Thus, the reduced reactivity to painful shocks we found, in prenatally stressed males, was not due to a lower sensitivity to shocks, but probably it was governed by other patterns of emotional behaviour, which could reflect differences in the activation threshold of some brain structures [32] [33]. On the other hand, SERT was effective in reversing these effects of the PS, possibly, by regulating the autonomic functions [34].

\subsection{Sex-Differences in Learning Performance and Escape Failure}

The exposure to IS prior to escapable shocks affected the learning performance and the shocks avoidance in a sex-dependent manner. According to the literature, subjecting rats to an uncontrollable traumatic event, such as the IS, disturbs their later escape performance [4] [17] [20] [35] [36]. It was reported that more than 95\% of male rats develop an escape deficit after they had been subjected to an inescapable stressful situation 24 hours earlier [35]. Normally, the LH paradigm uses a large number of high-intensity footshocks [20] or is applied over several consecutive days [17]. In the present study, the single mild footshock session we used was apparently sufficient to trigger the same deleterious effects in all the animals, except the prenatally stressed females. The PS females exhibited the greatest ability to learn to avoid the adverse stimuli, and did not display escape deficit.

During the first trials, when the rats were in the charged compartment, they did not enter the opposite chamber readily, as expected in this type of bi-directional training, even when is not preceded by IS [37]. In ensuing sessions, rats can either learn to avoid shocks-they learn by association that after light comes the shock, and that this can be avoided by crossing the gate-or they fail to learn, and receive repeated footshocks. In a previous experiment carried out at our laboratory, the avoidance behaviour was tested and $90 \%$ of the naïve male rats managed to achieve scores of $60 \%-80 \%$ in conditioning [38]. The data obtained in the present work reveal differences in the effects of PS on the learning performance and escape failure according to sex. Our results show that after being exposed to IS, whereas the males born from stressed mothers did not reach to learn, the PS females learned to escape from the shocks, as they were not affected by the previous IS. It could thus be suggested that the model of gestational stress we tested, helps females, at least regarding the ability to learn in a fear context. In males this was not the case. By contrast, the present data show that males subjected to PS were the most susceptible in developing the escape deficit, apart from being the only experimental group in which the animals exhibited an extreme behavioural response. Most PS males failed to respond to the adverse situation remaining still (while receiving the shocks), and they exhibited a significantly reduced interest in their environment; these symptoms can be attributed to the animal model of LH [15] [33] [35] [36] [39]. Importantly, and in contrast to males, most of the females did not exhibit this depressive-like state, regardless of previous stress. This agrees 
with the literature, whereas women are more susceptible than men to suffer from depression or post-traumatic stress disorder [40] [41], female rats rarely develop LH [17] [18].

Also, it was suggested that when tested on a learning or memory task context the better performance of females could be related to differences in their activity, in which motor function is required [32] [36] [42]. In the present study, the exposure to IS confirmed the sex-differences in escape behaviour and in the activity levels, but only if the females were subjected to PS. In the animals raised in standard conditions (Controls) no sex differences were found.

\subsection{Sex-Dependent Effects of Prenatal Stress on Learned Helplessness}

Helplessness behaviour and learning deficits in prenatally stressed animals have been reported previously [5] [8] [43]. With the PS paradigm, most authors have reported a greater immobility in the helplessness test in stressed animals and reduced memory retention on the passive avoidance tasks; and some differences according to sex have been noted. It has been described that female offspring appear to be relatively more resistant to stress exposure than males during development, the effects of PS affecting memory and learning being less evident in females [6] [44] [45].

Several studies reported that the differences between sexes, in the response to a fear context, arise from changes in the gonadal hormone levels during foetal development [6] [17] [46], persisting in gonadectomized adult animals [17]. Testosterone and estrogens could exert an active role, exacerbating or protecting the effects of the manipulation during pregnancy [47]. It is known that gestational stress reduces the level of testosterone in adults [45] and that low testosterone levels are associated with memory loss in men. This is consistent with our results showing disturbed behaviour in PS males that could be related to inadequate levels of testosterone, originally caused by the gestational stress. Considering that there are sex-dependent differences in the sensitivity to foetal programming [16], it is possible that during such critical brain development period, sex hormones give rise to sex differences with regards to learned helplessness-eliciting behaviours. Moreover, although the cause remains to be totally established, other authors also reported that PS may alter the ability of estrogens to alleviate some depressive behaviour in female but not male rats [47].

\subsection{The Effects of Sertraline}

In addition to the disturbance of serotoninergic metabolism by PS [48], subjecting rats to inescapable stressors also induces a decrease in the levels of 5-HT in some brain areas [20], and it is expected that chronic treatment with SERT, which increases the level of this neurotransmitter, would reverse such an effect. Our data show that the LH phenomenon was sensitive to the treatment with SERT. SERT was effective in reducing escape failure and in preventing males' hypoactivity.

Moreover, among non-stressed animals our data show that SERT was effective in reversing IS-induced learning deficits, but only in control females; in males, this was not the case. Although the existence of sex differences in responses to drugs is not remarkable, since these have been reported in both humans and rodents [32], and specifically in the response to SERT [49] [50], the effects of PS affecting drug effectiveness is more difficult to interpret. The animals escaped from the shocks, but did not avoid them. Many of these animals, which were previously stressed and were receiving SERT, though not despaired, did not manage to avoid the shocks. It was expected that the rats treated with SERT would improve their shocks avoidance. In a stressful situation, 5-HT should help to establish behavioural alterations in the most appropriate direction [51] [52]. Nevertheless, the present results strengthen past reports in the sense that learning is affected by drug treatment with serotonin precursors (e.g. 1-tryptophan and 5-HT) [16]. In such study the deficit in the acquisition of escape behaviour was only induced by the combination of exposing the animals to IS and the increase in 5-HT levels [16]. It was suggested that the animals would be slower due to alterations at the level of motor activity. It is known that in addition to its role in the regulation of emotions, 5-HT can also influence other functions such as sensory perception or motor activity [53]. However, we found no differences in the locomotor activity as effect of SERT administration. This supports previous results [13] [51] [54] in which fluoxetine or SERT reduced the stressinduced immobility, without influencing other activities.

Moreover, our data show an increase in most emotionality parameters in the SERT-taking animals. In response to an unexpected stress the dorsal area of the raphe nuclei (DRN) is activated, with the consequent release of 5-HT in the limbic area and subcortical structures [51]. Apparently, the activation of the DRN seroton- 
ergic neurons facilitates anxiety-like behavioural responses [55]. Thus, by increasing serotonin turnover in this circuitry, SERT treatment modulates depressive behaviours, but may have increased anxiety during each exposure to the stressful context. Despite this, it could be suggested that the improved anxiety in SERT-receiving animals might also be a consequence of not escaping from shocks. In contrast to the protocol of previous experiments [23], in the present work, the animals that did not acquire avoidance responses were exposed persistently to the shocks.

In light of the results, we surmise that the learning deficit exhibited by stressed animals receiving SERT would not be due to a deficit in perception, memory, pain or motor impairments. In fact, we did not find stimulus perception to be affected, and the rats did not find difficulties in associating the CS with the US. The rats looked at the light when this was on, and jumped and escaped when they were subjected to shocks, but for some reason they did not avoid them. Indeed, we observed that some animals from this experimental group exhibited a preparatory behavioural posture (data not shown), which would allow them to rapidly pass from one compartment to the other-they stand at the door-but even though they could escape they did not, waiting for the shock to pass. This behavioural posture had already been observed [37].

\section{Conclusion}

Our data agree with and extend previous reports based on the idea that exposure to PS affects subsequent behaviour and the response to stress, later in life, in a sex-dependent way. In this study, the males were more likely to develop behavioural despair than the females. It could be speculated that the functional connection between the forebrain and brainstem regions has been affected, as an effect of the early stress, in males but not in females, leading to a dysregulation of the stress response. When exposed to a fear context, oestrogen levels could have been modulating the behavioural and neurobiological responses, and somehow, protecting the effects of early stress in females. Further research is required to identify the neuronal mechanisms that mediate the differing responses, between males and females, to dramatic events and their recovery with antidepressants.

\section{Acknowledgements}

This research was supported by grants from the Spanish Ministry of Science and Innovation (MICINN, \#BFU2007-65210 and \#BFU2010-17754) to Dr. Dolores E. López and by the University of Salamanca Research Support Grant 2015 to Dr. Dolores E. López.

\section{References}

[1] Choy, K.H., de Visser, Y.P. and van den Buuse, M. (2009) The Effect of “Two Hit” Neonatal and Young-Adult Stress on Dopaminergic Modulation of Prepulse Inhibition and Dopamine Receptor Density. British Journal of Pharmacology, 156, 388-396. http://dx.doi.org/10.1111/j.1476-5381.2008.00008.x

[2] García-Cáceres, C., Lagunas, N., Calmarza-Font, I., Azcoitia, I., Diz-Chaves, Y., García-Segura, L.M., et al. (2010) Gender Differences in the Long-Term Effects of Chronic Prenatal Stress on the HPA Axis and Hypothalamic Structure in Rats. Psychoneuroendocrinology, 35, 1525-1535. http://dx.doi.org/10.1016/j.psyneuen.2010.05.006

[3] Fujioka, T., Fujioka, A., Tan, N., Chowdhury, G.M.I., Mouri, H., Sakata, Y., et al. (2001) Mild Prenatal Stress Enhances Learning Performance in the Non-Adopted Rat Offspring. Neuroscience, 103, 301-307. http://dx.doi.org/10.1016/S0306-4522(00)00582-0

[4] Leventopoulos, M., Russig, H., Feldon, J., Pryce, C.R. and Opacka-Juffry, J. (2009) Early Deprivation Leads to LongTerm Reductions in Motivation for Reward and 5-HT1A Binding and Both Effects Are Reversed by Fluoxetine. Neuropharmacology, 56, 692-701. http://dx.doi.org/10.1016/j.neuropharm.2008.12.005

[5] Alleva, E. and Francia, N. (2009) Psychiatric Vulnerability: Suggestions from Animal Models and Role of Neurotrophins. Neuroscience \& Biobehavioral Reviews, 33, 525-536. http://dx.doi.org/10.1016/j.neubiorev.2008.09.004

[6] Cherian, S.B., Bairy, K.L. and Rao, M.S. (2009) Chronic Prenatal Restraint Stress Induced Memory Impairment in Passive Avoidance Task in Post Weaned Male and Female Wistar Rats. Indian Journal of Experimental Biology, 47, 893-899.

[7] Darnaudéry, M. and Maccari, S. (2008) Epigenetic Programming of the Stress Response in Male and Female Rats by Prenatal Restraint Stress. Brain Research Reviews, 57, 571-585. http://dx.doi.org/10.1016/j.brainresrev.2007.11.004

[8] Abe, H., Hidaka, N., Kawagoe, C., Odagiri, K., Watanabe, Y., Ikeda, T., Ishizuka, Y., et al. (2007) Prenatal Psychological Stress Causes Higher Emotionality, Depression-Like Behavior, and Elevated Activity in the Hypothalamo- 
Pituitary-Adrenal Axis. Neuroscience Research, 59, 145-151. http://dx.doi.org/10.1016/j.neures.2007.06.1465

[9] Lehmann, J., Pryce, C.R. and Feldon, J. (2000) Lack of Effect of an Early Stressful Life Event on Sensorimotor Gating in Adult Rats. Schizophrenia Research, 41, 365-371. http://dx.doi.org/10.1016/S0920-9964(99)00080-8

[10] Oomen, C.A., Soeters, H., Audureau, N., Vermunt, L., van Hasselt, F.N., Manders, E.M., et al. (2010) Severe Early Life Stress Hampers Spatial Learning and Neurogenesis, but Improves Hippocampal Synaptic Plasticity and Emotional Learning under High-Stress Conditions in Adulthood. Journal of Neuroscience, 30, 6635-6645. http://dx.doi.org/10.1523/JNEUROSCI.0247-10.2010

[11] Archer, J.E. and Blackman, D.E. (1971) Prenatal Psychological Stress and Offspring Behavior in Rats and Mice. Developmental Psychobiology, 4, 193-248. http://dx.doi.org/10.1002/dev.420040302

[12] Gué, M., Bravard, A., Meunier, J., Veyrier, R., Gaillet, S., Recasens, M., et al. (2004) Sex Differences in Learning Deficits Induced by Prenatal Stress in Juvenile Rats. Behavioural Brain Research, 150, 149-157. http://dx.doi.org/10.1016/S0166-4328(03)00250-X

[13] Pereira-Figueiredo, I., Sancho, C., Carro, J., Castellano, O. and Lopez, D.E. (2014) The Effects of Sertraline Administration from Adolescence to Adulthood on Physiological and Emotional Development in Prenatally Stressed Rats of Both Sexes. Frontiers in Behavioral Neuroscience, 8, 260. http://dx.doi.org/10.3389/fnbeh.2014.00260

[14] Roussel, S., Boissy, A., Montigny, D., Hemsworth, P.H. and Duvaux-Ponter, C. (2005) Gender-Specific Effects of Prenatal Stress on Emotional Reactivity and Stress Physiology of Goat Kids. Hormones and Behavior, 47, 256-266. http://dx.doi.org/10.1016/j.yhbeh.2004.09.010

[15] Maier, S.F. (1984) Learned Helplessness and Animal Models of Depression. Progress in Neuro-Psychopharmacology Biological Psychiatry, 8, 435-446. http://dx.doi.org/10.1016/S0278-5846(84)80032-9

[16] Brown, L., Rosellini, R.A., Samuels, O.B. and Riley, E.P. (1982) Evidence for a Serotonergic Mechanism of the Learned Helplessness Phenomenon. Pharmacology Biochemistry and Behavior, 17, 877-883.

[17] Dalla, C., Edgecomb, C., Whetstone, A.S. and Shors, T.J. (2008) Females Do Not Express Learned Helplessness Like Males Do. Neuropsychopharmacology, 33, 1559-1569.

[18] Steenbergen, H.L., Heinsbroek, R.P., Van Hest, A. and Van de Poll, N.E. (1990) Sex-Dependent Effects of Inescapable Shock Administration on Shuttlebox-Escape Performance and Elevated Plus-Maze Behavior. Physiology \& Behavior, 48, 571-576. http://dx.doi.org/10.1016/0031-9384(90)90302-K

[19] West, C.H. and Weiss, J.M. (2005) A Selective Test for Antidepressant Treatments Using Rats Bred for Stress-Induced Reduction of Motor Activity in the Swim Test. Psychopharmacology (Berl), 182, 9-23. http://dx.doi.org/10.1007/s00213-005-0048-X

[20] Zazpe, A., Artaiz, I., Labeaga, L., Lucero, M.L. and Orjales, A. (2007) Reversal of Learned Helplessness by Selective Serotonin Reuptake Inhibitors in Rats Is Not Dependent on 5-HT Availability. Neuropharmacology, 52, 975-984. http://dx.doi.org/10.1016/j.neuropharm.2006.10.014

[21] Jourdan, D., Ardid, D., Chapuy, E., Eschalier, A. and Le Bars, D. (1995) Audible and Ultrasonic Vocalization Elicited by Single Electrical Nociceptive Stimuli to the Tail in the Rat. Pain, 63, 237-249. http://dx.doi.org/10.1016/0304-3959(95)00049-X

[22] Kosten, T.A., Miserendino, M.J.D., Bombace, J.C., Lee, H.J. and Kim, J.J. (2005) Sex-Selective Effects of Neonatal Isolation on Fear Conditioning and Foot Shock Sensitivity. Behavioural Brain Research, 157, 235-244. http://dx.doi.org/10.1016/j.bbr.2004.07.001

[23] Li, S., Wang, C., Wang, M.-W., Murakami, Y. and Matsumoto, K. (2006) Impairment of the Spatial Learning and Memory Induced by Learned Helplessness and Chronic Mild Stress. Pharmacology Biochemistry and Behavior, 83, 186-193. http://dx.doi.org/10.1016/j.pbb.2006.01.004

[24] Wakizono, T., Sawamura, T., Shimizu, K., Nibuya, M., Suzuki, G., Toda, H., et al. (2007) Stress Vulnerabilities in an Animal Model of Post-Traumatic Stress Disorder. Physiology \& Behavior, 90, 687-695. http://dx.doi.org/10.1016/j.physbeh.2006.12.008

[25] Alonso, S.J., Castellano, M.A., Afonso, D. and Rodriguez, M. (1991) Sex Differences in Behavioral Despair: Relationships between Behavioral Despair and Open Field Activity. Physiology Behavior, 49, 69-72. http://dx.doi.org/10.1016/0031-9384(91)90232-D

[26] Nederhof, E. and Schmidt, M.V. (2012) Mismatch or Cumulative Stress: Toward an Integrated Hypothesis of Programming Effects. Physiology Behavior, 106, 691-700. http://dx.doi.org/10.1016/j.physbeh.2011.12.008

[27] Blanchard, D.C., Shepherd, J.K., Carobrez, A.P. and Blanchard, R.J. (1991) Sex Effects in Defensive Behavior: Baseline Differences and Drug Interactions. Neuroscience \& Biobehavioral Reviews, 15, 461-468. http://dx.doi.org/10.1016/S0149-7634(05)80132-0

[28] Hillman, C.H., Hsiao-Wecksler, E.T. and Rosengren, K.S. (2005) Postural and Eye-Blink Indices of the Defensive 
Startle Reflex. International Journal of Psychophysiology, 55, 45-49. http://dx.doi.org/10.1016/j.ijpsycho.2004.06.002

[29] Duchesne, A., Dufresne, M.M. and Sullivan, R.M. (2009) Sex Differences in Corticolimbic Dopamine and Serotonin Systems in the Rat and the Effect of Postnatal Handling. Progress in Neuro-Psychopharmacology and Biological Psychiatry, 33, 251-261. http://dx.doi.org/10.1016/j.pnpbp.2008.11.012

[30] Sternberg, W.F. (1999) Sex Differences in the Effects of Prenatal Stress on Stress-Induced Analgesia. Physiology \& Behavior, 68, 63-72. http://dx.doi.org/10.1016/S0031-9384(99)00164-X

[31] Takahashi, L.K., Baker, E.W. and Kalin, N.H. (1990) Ontogeny of Behavioral and Hormonal Responses to Stress in Prenatally Stressed Male Rat Pups. Physiology \& Behavior, 47, 357-364. http://dx.doi.org/10.1016/0031-9384(90)90154-V

[32] Leuner, B., Mendolia-Loffredo, S. and Shors, T.J. (2004) Males and Females Respond Differently to Controllability and Antidepressant Treatment. Biological Psychiatry, 56, 964-970. http://dx.doi.org/10.1016/j.biopsych.2004.09.018

[33] Pittenger, C. and Duman, R.S. (2008) Stress, Depression, and Neuroplasticity: A Convergence of Mechanisms. Neuropsychopharmacology, 33, 88-109. http://dx.doi.org/10.1038/sj.npp.1301574

[34] Van den Hove, D.L.A., Leibold, N.K., Strackx, E., Martinez-Claros, M., Lesch, K.P., Steinbusch, H.W.M., et al. (2014) Prenatal Stress and Subsequent Exposure to Chronic Mild Stress in Rats; Interdependent Effects on Emotional Behavior and the Serotonergic System. European Neuropsychopharmacology, 24, 595-607. http://dx.doi.org/10.1016/j.euroneuro.2013.09.006

[35] Mangiavacchi, S., Masi, F., Scheggi, S., Leggio, B., De Montis, M.G. and Gambarana, C. (2001) Long-Term Behavioral and Neurochemical Effects of Chronic Stress Exposure in Rats. Journal of Neurochemistry, 79, 1113-1121. http://dx.doi.org/10.1046/j.1471-4159.2001.00665.x

[36] Padilla, E., Barrett, D., Shumake, J. and Gonzalez-Lima, F. (2009) Strain, Sex, and Open-Field Behavior: Factors Underlying the Genetic Susceptibility to Helplessness. Behavioural Brain Research, 201, 257-264. http://dx.doi.org/10.1016/j.bbr.2009.02.019

[37] Savonenko, A.V., Brush, F.R. and Zielinski, K. (1999) How Do Rats Cope with the Two-Way Escape Problem in a Homogeneous Shuttle Box? Acta Neurobiologiae Experimentalis (Wars), 59, 145-157.

[38] Castellano, O., Moscoso, A., Riolobos, A.S., Carro, J., Arji, M., Molina, V., et al. (2009) Chronic Administration of Risperidone to Healthy Rats: A Behavioural and Morphological Study. Behavioural Brain Research, 205, 488-498. http://dx.doi.org/10.1016/j.bbr.2009.08.002

[39] El Yacoubi, M., Bouali, S., Popa, D., Naudon, L., Leroux-Nicollet, I., Hamon, M., et al. (2003) Behavioral, Neurochemical, and Electrophysiological Characterization of a Genetic Mouse Model of Depression. Proceedings of the National Academy of Sciences of the United States of America, 100, 6227-6232. http://dx.doi.org/10.1073/pnas.1034823100

[40] Holbrook, T.L., Hoyt, D.B., Stein, M.B. and Sieber, W.J. (2002) Gender Differences in Long-Term Posttraumatic Stress Disorder Outcomes after Major Trauma: Women Are at Higher Risk of Adverse Outcomes than Men. The Journal of trauma, 53, 882-888. http://dx.doi.org/10.1097/00005373-200211000-00012

[41] Seney, M.L. and Sibille, E. (2014) Sex Differences in Mood Disorders: Perspectives from Humans and Rodent Models. Biology of Sex Differences, 5, 17. http://dx.doi.org/10.1186/s13293-014-0017-3

[42] Shors, T.J., Mathew, J., Sist,i H.M., Edgecomb, C., Beckoff, S. and Dalla, C. (2007) Neurogenesis and Helplessness Are Mediated by Controllability in Males But Not in Females. Biological Psychiatry, 62, 487-495. http://dx.doi.org/10.1016/j.biopsych.2006.10.033

[43] Drago, F., Di Leo, F. and Giardina, L. (1999) Prenatal Stress Induces Body Weight Deficit and Behavioural Alterations in Rats: The Effect of Diazepam. European Neuropsychopharmacology, 9, 239-245. http://dx.doi.org/10.1016/S0924-977X(98)00032-7

[44] Zueña, A.R., Mairesse, J., Casolini, P., Cinque, C., Alema, G.S., Morley-Fletcher, S., et al. (2008) Prenatal Restraint Stress Generates Two Distinct Behavioral and Neurochemical Profiles in Male and Female Rats. PLoS ONE, 3, e2170. http://dx.doi.org/10.1371/journal.pone.0002170

[45] Van den Hove, D.L.A., Kenis, G., Brass, A., Opstelten, R., Rutten, B.P.F., Bruschettini, M., et al. (2012) Vulnerability versus Resilience to Prenatal Stress in Male and Female Rats; Implications from Gene Expression Profiles in the Hippocampus and Frontal Cortex. European Neuropsychopharmacology, 23, 1226-1246. http://dx.doi.org/10.1016/j.euroneuro.2012.09.011

[46] Ter Horst, G.J., Wichmann, R., Gerrits, M., Westenbroek, C. and Lin, Y. (2009) Sex Differences in Stress Responses: Focus on Ovarian Hormones. Physiology \& Behavior, 97, 239-249. http://dx.doi.org/10.1016/j.physbeh.2009.02.036

[47] Frye, C.A. and Wawrzycki, J. (2003) Effect of Prenatal Stress and Gonadal Hormone Condition on Depressive Behaviors of Female and Male Rats. Hormones and Behavior, 44, 319-326. http://dx.doi.org/10.1016/S0018-506X(03)00159-4 
[48] Maccari, S. and Morley-Fletcher, S. (2007) Effects of Prenatal Restraint Stress on the Hypothalamus-Pituitary-Adrenal Axis and Related Behavioural and Neurobiological Alterations. Psychoneuroendocrinology, 32, S10-S15. http://dx.doi.org/10.1016/j.psyneuen.2007.06.005

[49] Pereira-Figueiredo, I., Carro, J., Muñoz, L.J., Sancho, C., Castellano, O., Gómez-Nieto, R. and López, D.E. (2015) Sex Differences in the Effects of Sertraline and Stressors in Rats Previously Exposed to Restraint Stress. Journal of Biomedical Science and Engineering, 8, 399-419. http://dx.doi.org/10.4236/jbise.2015.87038

[50] Broadbear, J.H., Nguyen, T., Clarke, I.J. and Canny, B.J. (2004) Antidepressants, Sex Steroids and Pituitary-Adrenal Response in Sheep. Psychopharmacology (Berl), 175, 247-255. http://dx.doi.org/10.1007/s00213-004-1811-0

[51] Maier, S.F. and Watkins, L.R. (2005) Stressor Controllability and Learned Helplessness: The Roles of the Dorsal Raphe Nucleus, Serotonin, and Corticotropin-Releasing Factor. Neuroscience \& Biobehavioral Reviews, 29, 829-841. http://dx.doi.org/10.1016/j.neubiorev.2005.03.021

[52] Nowakowska, E., Kus, K., Chodera, A. and Rybakowski, J. (2000) Behavioural Effects of Fluoxetine and Tianeptine, Two Antidepressants with Opposite Action Mechanisms, in Rats. Arzneimittel-Forschung, 50, 5-10.

[53] Jeffrey Newport, D., Wilcox, M.M. and Stowe, Z.N. (2001) Antidepressants during Pregnancy and Lactation: Defining Exposure and Treatment Issues. Seminars in Perinatology, 25, 177-190. http://dx.doi.org/10.1053/sper.2001.24901

[54] Bilge, S., Bozkurt, A., Bas, D.B., Aksoz, E., Savli, E., Ilkaya, F., et al. (2008) Chronic Treatment with Fluoxetine and Sertraline Prevents Forced Swimming Test-Induced Hypercontractility of Rat Detrusor Muscle. Pharmacological Reports, 60, 872-879.

[55] Hale, M.W., Raison, C.L. and Lowry, A. (2013) Integrative Physiology of Depression and Antidepressant Drug Action: Implications for Serotonergic Mechanisms of Action and Novel Therapeutic Strategies for Treatment of Depression. Pharmacology \& Therapeutics, 137, 108-118. http://dx.doi.org/10.1016/j.pharmthera.2012.09.005 